\title{
BM] Global Health WHO Multi-Country Survey on Abortion-related Morbidity and Mortality in Health Facilities: study protocol
}

\author{
Caron R Kim, Özge Tunçalp, Bela Ganatra, Ahmet Metin Gülmezoglu, \\ WHO MCS-A Research Group
}

To cite: Kim CR,

Tunçalp Özge, Ganatra B, et al. WHO Multi-Country Survey on Abortion-related Morbidity and Mortality in Health Facilities:

study protocol. BMJ Global Health 2016;1:e000113. doi:10.1136/bmjgh-2016000113

Received 22 June 2016 Revised 19 August 2016 Accepted 30 August 2016

CrossMark

UNDP/UNFPA/UNICEF/WHO/ World Bank Special Programme of Research, Development and Research Training in Human Reproduction (HRP), Department of Reproductive Health and Research, WHO, Geneva, Switzerland

Correspondence to Dr Caron R Kim; kimca@who.int

\section{ABSTRACT}

Introduction: According to the WHO, abortion accounts for about $8 \%$ (4.7-13.2) of maternal mortality worldwide. In 2010, the WHO MultiCountry Survey (MCS) on Maternal and Newborn Health collected data on over 300000 women who were admitted in health facilities to receive pregnancy-related care. Abortion data were partially captured by centring on severe maternal outcomes (ie, near-miss or maternal deaths). Building on the experiences of the prior MCS as well as current WHO reproductive health projects, we are undertaking a multi-country survey to better capture the burden and severity of abortion-related complications and management among women presenting to the health facilities.

Methods and analysis: This is a large crosssectional study with prospective data collection. It will be implemented in health facilities in 30 countries across the WHO regions of Africa, Americas, Eastern Mediterranean, Europe, South East Asia and Western Pacific. Countries and facilities will be identified through a multistage sampling methodology. Data collection will be at both the facility and individual levels, involving review of medical records and exit surveys with eligible women using audio computer-assisted selfinterview. All women presenting to the health facilities with signs and symptoms of abortion complications will comprise the study population.

Online data entry and management will be performed on a web-based data management system. Analysis will include prevalence of abortionrelated complications and descriptive frequencies of procedural/non-procedural management and experience of care.

Ethics and dissemination: Ethical issues of the consent process are addressed. Dissemination plans will involve the participating facilities and communities to further strengthen abortion-related research capacity within the MCS on Abortion (MCSA) countries. Furthermore, dissemination of results will be an iterative process at both the facility and national level to potentially propagate positive changes to abortion-related policies and practices.

\section{Key questions}

What is already known about this topic?

- Capturing accurate information on abortion is a challenge especially in settings where abortion is legally restricted.

- As deaths resulting from unsafe abortion have decreased in recent years, the focus is shifting towards complications associated with abortion.

\section{What are the new findings?}

- This study will provide new information by assessing the burden, severity and management of complications due to abortion in a standardised way in the health facilities in 30 countries.

- Findings will also include the experience of care women with abortion-related complications received at these facilities.

Recommendations for policy

- Findings from this study offer potential recommendations for policy and practice that support the provision of safe abortion.

\section{INTRODUCTION}

According to WHO abortion accounts for about $8 \%$ (4.7-13.2) of maternal mortality worldwide. ${ }^{1}$ The term 'abortion' has become synonymous with induced abortion which is an intentional termination of pregnancy but it also includes spontaneous abortion, also known as miscarriage. Maternal deaths due to abortion, and more specifically unsafe induced abortion, is associated with a risk of misclassification, which might lead to underreporting. ${ }^{2}$ Legal, social and cultural ramifications that are associated with abortion mean that women are reticent to disclose abortion attempts and relatives or healthcare professionals tend to not report deaths as such. ${ }^{1}$ Moreover, as deaths resulting from unsafe abortion have decreased in recent 
years, the focus is shifting towards adverse outcomes associated with abortion. ${ }^{3}$ By analysing the national estimates of abortion-related hospital admissions for 26 developing countries, it is estimated that seven million women were treated for complications from unsafe pregnancy termination in $2012 .{ }^{4}$

The studies that look at the morbidity of abortion are limited and varied. These studies offer a quantification of the abortion-related outcomes, but they often do not investigate the nature or severity of the complications. ${ }^{5-9}$ There are only a few studies that offer such details of the abortion complications, with varying categorisations; ${ }^{10-15}$ these same studies have recommended a systematic definition of the severity of complications, in line with a systematic review which assessed the types and frequencies of complications. As a result of this analysis, the review highlighted the need for standardised definitions for future studies to help quantify the global burden. ${ }^{16}$ A follow-up systematic review proposed the use of the near-miss concept as a standardised approach to measure very severe, life-threatening conditions resulting from abortion. ${ }^{17}$ Since this review, this approach has been used in several studies. ${ }^{18} 19$ WHO identifies a near miss as 'a woman who nearly died but survived a complication that occurred during pregnancy, childbirth or within 42 days of termination of pregnancy', allowing a standardised approach to measuring very severe morbidity using clinical, laboratory and management-based markers. ${ }^{20}$ The less severe complications which are the conditions that do not fall under the near-miss category yet are complications that warranted medical care, remains to be better delineated.

Using this maternal near-miss approach in 2011-2012, the WHO Multi-Country Survey (WHO MCS) on Maternal and Newborn Health collected data on over 300000 women who received pregnancy-related care in 359 facilities across 29 countries. ${ }^{21}$ A secondary analysis of WHO MCS was performed to analyse the data on women with severe maternal outcomes related to abortion who sought care at the participating health facilities. ${ }^{22}$ Several limitations were highlighted. First, there was variance among facilities in their reporting of abortion cases-the most likely reason being that severe abortion-related morbidity and mortality was not the primary focus of the WHO MCS. There was also no distinction in the circumstances of the woman's pregnancy termination. It was unclear to whether the woman had experienced a spontaneous or an induced abortion given that the clinical signs and symptoms are similar and that under-reporting by the woman would be a major factor to this situation.

To fully explore management of complications of abortion, this study will connect to current WHO projects. A WHO observational study in five countries (Ghana, Laos, Myanmar, Nigeria and Sri Lanka) is providing a better understanding of the relationship between misoprostol use and the type and severity of abortion symptoms. The study is also documenting the care-seeking behaviour of women. ${ }^{23}$ The review of studies that informed this five-country study included studies in which data were obtained via medical records followed by an interview of the patients or through a questionnaire. ${ }^{24-29}$ Including direct reporting from women allowed for a more accurate assessment of the patient's diagnosis and ability to obtain sensitive information that would be harder to determine through the medical records.

Asking women about their experiences coupled with the medical data on the management of complications allows exploration to the overall quality of care women are receiving. WHO has been looking closely at the concept of quality of care and how it goes beyond provision of care by incorporating experience of care. ${ }^{30}$ Domains that make up the 'experience of care' include effective communication, respect and dignity and emotional support. ${ }^{31} 32$ Therefore, while assessing the quality of care for women with abortion complications, it is important to explore how women experienced the care they received in these facilities as well as to investigate management of abortion complications.

In addition to inquiring about the quality of care, exploration of abortion safety is crucial. Discussions revolving around what constitutes 'abortion safety' have given insight into the changing nature of the content of this concept. The WHO definition of unsafe abortion is any procedure terminating a pregnancy that is performed by persons lacking in the necessary skills and/or in an environment not in conformity with minimal medical standards. ${ }^{33}$ However, this definition is dynamic and evolves with the evidence-based WHO recommendations related to method, provider and setting based on the gestational age. ${ }^{34}$ For example, WHO guidelines recommend vacuum aspiration rather than sharp curettage which had been the method used previously. In addition, self-induced abortion is still prevalent with the persistence of traditional methods being used ${ }^{35} 36$ and information on misoprostol being widespread. Therefore, it has been recommended to interpret safe abortion in line with the current WHO technical and policy guidance.

Linking with the aforementioned ongoing projects and building on the existing literature and the WHO MCS network, we are undertaking a multi-country survey specifically focusing on abortion to obtain a better picture of the abortion-related morbidity and mortality globally. This survey will give us an opportunity to assess the burden, severity and management of complications due to abortion in a standardised way in the facilities across 30 countries and explore how women experience the care they receive in these facilities. ${ }^{37}$

\section{Objectives}

In this manuscript, we present the methods of the WHO MCS on Abortion-related Morbidity and Mortality. The main objectives of this study are to assess the frequency 
and severity of abortion-related complications, to determine management of these complications, to determine the conditions (location, provider, management provided as per gestational age) under which the abortions that led to complications took place and to explore the experience of care women with abortion-related complications received at these facilities. Secondary objectives include assessing the institutional capacity for provision of safe abortion management of complications, exploring the outcomes of early pregnancy losses other than abortion such as ectopic and molar pregnancies and to strengthen abortion-related research capacity within the participating countries.

\section{METHODS AND ANALYSIS}

This is a large cross-sectional study with prospective data collection at the facilities. It will be implemented in health facilities in 30 countries across the WHO regions of Africa, Americas, Eastern Mediterranean, Europe, South East Asia and Western Pacific. Multistage sampling will be used to identify countries, provinces/states and health facilities (figure 1). Some of these facilities may have previously been included in the WHO Global Survey and MCS. ${ }^{38} 39$ A facility assessment form will aid in the selection of the facilities that will be included in the survey for individual data collection. Once the appropriate facilities are selected, data collection will be conducted using medical records and exit surveys among women who are receiving care for abortion-related complications during the study period.

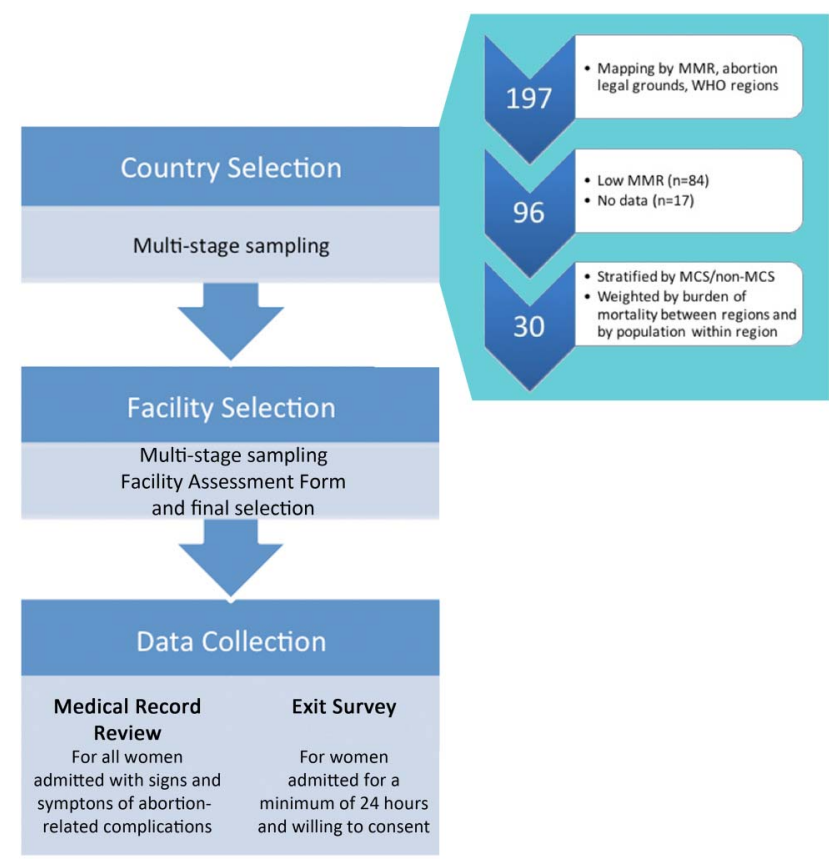

Figure 1 Overview of study design for WHO MCS-A. MMR, maternal mortality ratio; WHO MCS-A, WHO Multi-Country Survey on Abortion.

\section{Country and facility selection}

The first step of the multistage sampling is selection of the countries, summarised in figure 1. Initially the countries were mapped by their maternal mortality ratio (MMR) levels, abortion legal grounds and WHO regions. ${ }^{40}{ }^{41}$ The countries with low MMR, defined as MMR $<50$ based on most recent maternal mortality estimates ${ }^{40}$ and countries with no data were removed. The remaining countries were those with medium and high MMR and we performed random sampling from this pool. We also stratified the countries by their participation in the previous WHO MCS so that once random sampling resulted, we could include both a sample from new countries and prior MCS countries. As a result, 30 countries were randomly sampled, weighted by burden of maternal mortality between regions and by population within the region.

Based on this sampling strategy, the following countries have been identified: Algeria, Argentina, Bangladesh, Benin, Bolivia, Brazil, Burkina Faso, Chad, Cuba, Democratic Republic of the Congo, Dominican Republic, El Salvador, Ethiopia, Ghana, Guinea-Bissau, India, Indonesia, Kenya, Malawi, Morocco, Mozambique, Myanmar, Nepal, Niger, Nigeria, Pakistan, Peru, Philippines, Tajikistan and Uganda. For logistical (management of the implementation across countries including data quality assurance), financial and operational reasons based on the previous WHO MCS on caesarean section and maternal near miss, the study will be implemented in three phases. The phased implementation will start with the African countries, followed by the countries in the regions of Southeast Asia, Mediterranean and Western Pacific and end with the countries from the Americas Region.

After the selection of the study countries, the second stage of sampling will consist of random selection of two provinces/states, with probability proportional to the population size, plus the capital city/metropolitan area. For very large province/states, a third stage sampling will be used for sampling subdivisions below the province/state level. For very large cities, a fourth sampling stage will be implemented based on the random selection of city geographical subdivisions, with probability proportional to the respective populations. ${ }^{38}$

Once the geographical areas are selected, 10 facilities per state/province (with a total of 30 facilities per country) will be selected from the census of private and public facilities within that area fulfilling the following characteristics: facilities with $>1000$ deliveries per year, a gynaecology ward and surgical capability, defined as providing the signal functions for comprehensive emergency obstetric care, which includes removal of retained products and surgical capability. ${ }^{42}$ Facilities that have $<10$ postabortion care (PAC) patients reported over 1 month will be excluded.

If there are $<10$ facilities fulfilling these criteria, all eligible facilities in that area will be selected. In the case of a sampled facility refusing participation, it will be replaced by another facility. Prior use of this sampling 
scheme in the WHO Global Survey and MCS showed that such a sampling scheme is feasible and represents the facility-based healthcare systems available in the countries and applicable to our study topic of interest.

Sample size: There is considerable variation in reporting for abortion-related morbidity across facilities. ${ }^{16} 17$ Using individual facility data from a morbidity study on abortion conducted in Zambia, ${ }^{43}$ we have applied our facility sampling criteria of $>1000$ annual deliveries and surgical capability and calculated different scenarios for sample size and data collection period (table 1). These calculations are based on having at least 16 facilities per country. The lower limit presents the calculations excluding the biggest contributing facility in the sample. In our sampling design, we have planned for a maximum of 30 facilities per country.

Based on this, we conservatively estimate that each study country will have between 800 and 1000 women meeting the eligibility criteria for inclusion, making them eligible for medical data extraction. Therefore, the data collection period will be 3 months to account for variability across facilities and countries. Based on this, we also added the exclusion criterion for facilities that have $<10$ PAC patients reported over 1 month.

\section{Study participants}

After selection of the facilities, individual data collection will be conducted at the selected facilities during the study period. Data collection at the facilities will be obtained through two different resources at the individual level: (1) medical records, (2) exit surveys with women. For the medical record review component, all women admitted to the participating facilities with signs and symptoms of abortion-related complications will be included in the medical record review. This will include those women who present or are diagnosed during the hospital visit with an ectopic pregnancy or molar pregnancy. We also will record those cases of women who died from abortion-related complications. Exclusion criteria will include pregnant women with a diagnosis of threatened abortion which is defined as vaginal bleeding with a closed cervix. ${ }^{44}$ The criterion of all women presenting to the facilities rather than admission to the facility is used to avoid exclusion of those women who seek care in the facilities for less severe complications.

Table 1 Sample size calculation scenarios based on Zambia data from 16 facilities $^{43}$

\begin{tabular}{|llll}
\hline $\begin{array}{l}\text { Data } \\
\text { collection } \\
\text { period } \\
\text { (months) }\end{array}$ & $\begin{array}{l}\text { Number of } \\
\text { PAC } \\
\text { cases }\end{array}$ & $\begin{array}{l}\text { Number of } \\
\text { hospitalised } \\
\text { cases } \\
(\mathbf{2 4} \text { hours) }\end{array}$ & $\begin{array}{l}\text { Number of } \\
\text { hospitalised } \\
\text { near-miss } \\
\text { cases }\end{array}$ \\
\hline 1 & $658-997$ & $317-383$ & $40-67$ \\
\hline 2 & $1316-1973$ & $634-766$ & $80-134$ \\
\hline 3 & $1973-2990$ & $951-1148$ & $119-201$ \\
\hline 4 & $2631-3987$ & $1268-1531$ & $159-268$ \\
\hline
\end{tabular}

PAC, postabortion care.
The eligibility criteria for the exit survey component are women with abortion-related complications who are admitted for a minimum of 24 hours and able and willing to consent. This will allow us to include women with more severe complications, which are more likely to be due to induced abortion and explore the characteristics of the abortion and experience of care they received. If a woman was referred and/or died we will not be able to include her in the exit survey component, and this might be a potential source of bias; however, we note that we will report on the women who died or who were referred throughout the study.

\section{Data collection and data management}

Each region will have a regional coordinator. The regional study coordinators will oversee the implementation of the study and coordinate between the Steering Group and country principal investigators (PIs) throughout this process. For each country, country teams will be established. Each country will have a PI who will oversee the implementation of the study in the participating facilities and ensure compliance to the study protocol. For the study sites, each facility will have a hospital coordinator. In most cases, they will be the obstetrician/ gynaecologist at that facility. The role of this coordinator is to train the research assistants, supervise the data extraction and serve as the focal person for that facility and maintain communication with the country investigator(s). In each hospital, the hospital coordinator will identify and train one research assistant who is legally allowed to access medical records within the facility for data extraction.

The facility assessment form will be completed by the facility administrator or obstetrician/gynaecologist at each identified facility. This survey will include sections on the location and type of facility, structure and capacity, identification of morbidity and near miss, provision of abortion and management of complications. This form will aim to identify the actual burden related to abortion complications in each facility and will include questions on the numbers of PAC provision, gynaecology admissions due to PAC, manual vacuum aspiration and surgical evacuation at three different time points (over the past 30 days, past 3 months, typical month) to get a better idea of the capacity and the number of patients managed. The numbers reported from these facilities will inform the selection of facilities for the individual data collection.

At the individual level, the first step involves assessment of the medical records to include potentially all women who have been cared for at the participating facilities with signs and symptoms of abortion-related (spontaneous or induced) complications. The research assistants will be trained in identifying from the medical records the signs and symptoms of abortion-related complications. They will extract data from medical records of women whose care has been completed into case report forms developed for the study. One week of 
training and piloting of the tools and the data collection process will take place at each site for the research team, prior to the initiation of the study.

For the exit survey, eligible women will be approached and invited to participate in the exit interview after being cleared for discharge or are in stable condition. Study procedures will be explained and informed consent will be obtained. Questions in the survey will include brief sociodemographic background, gestational age, type of abortion, if induced, abortion safety characteristics (method used, provider, setting), and experience of care related to effective communication, respect and dignity and emotional support. With the help of the WHO framework on quality of care, ${ }^{31}$ objectives measures have been taken into account to reflect the woman's experience of care.

Exit surveys will be conducted at a private location with a focus on maintaining participant confidentiality. Audio computer-assisted self-interviewing (ACASI) will be used to further promote confidentiality. With this system, the participant listens to a recorded voice and responds to the question using the keyboard, mouse or touchscreen. The computer-assisted self-interview has been seen as a promising tool to measure sensitive or socially unacceptable behaviours. ${ }^{45} 46$ The ACASI adds the audio component which allows inclusion of illiterate participants. This technology also removes the interviewer bias and standardises question administration. ${ }^{47}$ This form of questionnaire has been used and accepted in survey research on several sensitive topics including abortion. ${ }^{48-50}$

A central system will be used for online data entry and management by the data management unit at Centro Rosarino de Estudios Perinatales (CREP) which is been responsible for data management of WHO Global Survey and MCS. The ACASI data will be stored in a secure file on the computer and the research teams will transfer these files at regular intervals to the central system managed by CREP. Data analysis and interpretation will be performed by the Project Steering Group in collaboration with the country teams.

\section{Data analysis}

As the study is following phased implementation by regions, data will be analysed by regions and then an overall analysis will be conducted once the implementation of the study is completed in all of the regions. By analysing data of each region, we are aiming to interpret the results to identify and respond to country-level and regional-level needs and priorities to better inform future steps. In addition, phased implementation will provide an opportunity to interpret the country and regional results more in depth than what can be done in an overall analysis.

It should be noted that for the women where we have information from the medical record review form and the exit surveys, the data will be linked and analysed accordingly. The main purpose of linking the clinical records and exit survey is to use the background information collected in the medical record review for the analysis of the ACASI data. This approach will allow us to have enriched data on each of these women by providing expanded information from the record review and ACASI interview. During the data analysis phase, we will identify the patterns (if any) and explore the distribution of missing data before we decide on the best method of analysis. Analysis techniques for a stratified multilevel sampling design will be used to obtain descriptive data on abortion-related complications at the facility, country and regional levels.

The general analytical approach for the main objectives is as follows:

- The prevalence of abortion-related complications presenting to the facilities will be determined for the following denominators: live births, deliveries, all gynaecology admissions. Proportion of women who are admitted to the gynaecology or intensive care unit wards for abortion complications, for whom a procedure to manage an abortion complication is performed, deaths and severe complications (ie, nearmiss and potentially life-threatening complications) due to abortion complications will also be assessed:

- The clinical criteria will be followed: medical records of all women who were cared for in the participating facilities with signs and symptoms of abortion-related (spontaneous or induced) complications will consist of the study population for the medical record review. Exclusion criteria will include pregnant women with diagnosis of threatened abortion which is defined as vaginal bleeding with a closed cervix. ${ }^{44}$ Another exclusion criterion will be women presenting to the facility for unrelated issues but whose medical records indicate a previous history of postabortion complications.

- Near-miss criteria: WHO identification criteria for maternal near-miss cases will be used. These nearmiss clinical indicators are signs of organ dysfunction based on clinical signs, laboratory markers and management-based proxies. ${ }^{51}$

- For less severe complications, gradation of the complication severity will be determined using the data collected on vital signs, physical examination and management strategies. Use of the maternal morbidity matrix $^{52}$ will elucidate the less severe complications.

- Descriptive frequencies of the use of procedural and non-procedural management interventions will be calculated (ie, use of vacuum aspiration vs dilation and curettage, use of parenteral antibiotics).

- Based on the abortion safety characteristics reported by the women, descriptive frequencies will be calculated for the method used, provider and setting and association of these characteristics with the morbidity and mortality and management.

- Women-reported measures of experience of care will be assessed and associations with the abortion characteristics, morbidity and management will be explored in multilogistic regression models. 


\section{Ethical considerations}

There are several ethical considerations to take into account for such a study. Unless it is recorded in the medical forms, the study will not try to differentiate between induced or spontaneous abortions. We hypothesise that women with complications who stay in the facility for longer periods of times and/or are admitted are more likely to have induced abortions; however, due to legal issues in a number of countries and the sensitive nature of this topic, we did not want to place the burden on the women nor on the research assistants and healthcare providers to specifically identify women with induced abortions. If it is recorded as such in the medical forms, this information will be extracted.

With regard to the consent process for the medical record review, individual consent will not be necessary due to the fact that this is an observational study in which data will be collected and extracted from the health facility medical records without identifying the study participants. However, as highlighted in the Sarkar, ${ }^{53}$ certain principles can be followed to help the ethical conduct of the study where a waiver of informed consent is granted. These four principles are the following: (1) only routine clinical information is used for the analysis; (2) any identifying information is removed from the data set by the data controllers (the hospital coordinator in our case) and the data are coded into alphanumeric format; (3) confidentiality clauses are explicitly specified for those conducting the data extraction (research assistants); and (4) finally, obtaining clearance from the Institutional Review Board. We have followed all four principles with our core protocol.

Although we are not seeking informed consent for the medical record review, each woman will have the opportunity to 'opt-out'. In each facility, we will place posters and information materials that the facility is engaged in research studies that involve medical record reviews. There will be a statement confirming confidentiality and that all records will be de-identified. At the end of the message, it will note that the patients can inform their provider if they want to 'opt-out' of these medical record studies. Care will be taken to place the information in areas visible to the women. In addition, the posters and information materials will be developed in the local languages. Providers will also be trained to convey the opt-out option at the start of the visit so that illiterate women will also understand their options.

The ACASI exit survey component will require informed consent. Potential participants for the exit survey will be fully informed of the study by the research assistant at the time of screening. Participation in the exit survey will be entirely voluntary and participants are free to refuse or leave at any time. Full informed consent will be ensured through the information and consent form, which will be available in the relevant local language. Sufficient time will be given for questions or clarifications. Participant information will remain confidential at all times. If the exit survey elicits any negative emotions or distress, an on-site counsellor will be available in addition to provision of additional information for resources outside of the facility.

Since our criteria focuses on women of reproductive age experiencing symptoms of abortion-related complications, this might invariably include minors. If this is the case, the informed consent process for the minor will follow the country and local policy regulations. If the minor is able to consent for herself and desires to participate, she will be included. If the country regulation includes informing a third party and the minor is willing for this, we will proceed with the enrolment process. If the minor does not desire informing a third party, then she will not be invited to participate.

In all participating countries, the relevant ethical clearance will be obtained. As a facility-based study, an authorisation to perform the study will be obtained at the institutional level from the responsible authority in all selected health facilities.

\section{Dissemination}

Findings from this study will act as a springboard to further investigations of abortion and strengthen the research capacities of the participating countries as well as inform abortion-related policy and practice. The results of this study by phases will be published in peerreviewed journals. Collaborating investigators will disseminate local and collective results to their department and authorities. Dissemination of results to participating institutions and communities will take place through meetings of stakeholders within the facilities and the communities. Local partners will be encouraged to use the data in their country settings. In addition, the ACASI interview techniques will provide new and original data on the care received at these participating facilities.

It is anticipated that the dissemination of study findings will help improve abortion care in several respects. The survey instruments will offer an informative process that can potentially effect changes in the facilities. For example, the individual medical record form inquires on the management strategy of the complications to see if it aligns with WHO recommendations. For those cases that are not following the recommended treatment, this may guide the managers to improve their abortion services. In addition, the use of ACASI will provide information on the quality of care received at the facility. Use of these tools is both for research purposes and for facilities in general to use the tools and the information gained to track and improve quality of care provided to women who seek care for abortion-related complications. Learning more about the situation in these countries and the regions in general could be used by the researchers, advocates and policymakers to encourage governments to re-examine their abortion laws and practices, and initiate changes that support the provision of safe abortion.

Handling editor Soumitra Bhuyan 
Collaborators WHO MCS-A Research Group: Listed in random order: Hailemichael Gselassi Ganania, Ipas Africa Alliance, Nairobi, Kenya; Seni Kouanda, Institut de Recherche en Science de la Sante, Ouagadougou, Burkina Faso; Cassimo Bique, Department of Obstetrics and Gynaecology, Faculdade de Medicina, Universidade Eduardo Mondlane, Maputo, Mozambique; Veronique Filippi, Faculty of Epidemiology and Population Health, London School of Hygiene and Tropical Medicine, London, UK; Daniel Giordano, Centro Rosarino de Estudios Perinatales, Rosario, Argentina; Clementine Rossier, University of Geneva, Geneva, Switzerland; Luis Gadama, College of Medicine, University of Malawi, Blantyre, Malawi; Sourou Béatrice Goufodji Kèkè, Centre de Recherche en Reproduction Humaine et en Démographie, Cotonou, Bénin; Nafissa Osman, Department of Obstetrics and Gynaecology, Faculdade de Medicina, Universidade Eduardo Mondlane, Maputo, Mozambique; Nafiou Idi, Faculte des Sciences de la Sante, Niamey, Niger; Zahida Qureshi, Department of Obstetrics and Gynaecology, University of Nairobi, Nairobi, Kenya; Philip Guest, Institute for Population and Social Research, Mahidol University Salaya, Nakhon Pathom, Thailand; Sally Griffin, International Centre for Reproductive Health-Mozambique, Maputo, Mozambique; Jean-Jose Wolomby-Molondo, Department de Gynecologie et Obstetrique, Cliniques, Universitaires de Kinshasa, Kinshasa, DRC; Rodolfo Gomez Ponce de Leon, CLAP/ PAHO/WHO Montevideo Uruguay; Joao Paulo Souza, UNDP/UNFPA/UNICEF/WHO/World Bank Special Programme of Research, Development and Research Training in Human Reproduction (HRP), Department of Reproductive Health and Research, WHO, Geneva, Switzerland; $R$ Marie Charlemagne Ouedraogo, Obstetrics and Gynecology of Universities, Ouagadougou, Burkina Faso; Scott Sughrue, Department of Public Health \& Community Medicine, Tufts University School of Medicine, Boston, USA; Rajani Ved, National Health Systems Resource Center, New Delhi, India; Ausbert Msusa, College of Medicine, University of Malawi, Blantyre, Malawi; Ndema Habib, UNDP/UNFPA/UNICEF/WHO/World Bank Special Programme of Research, Development and Research Training in Human Reproduction (HRP), Department of Reproductive Health and Research, WHO, Geneva, Switzerland; Katherine Ba-Thike, University of Medicine 1, Yangon, Myanmar; Thierry Madjadoum, Department of Obstetrics and Gynaecology, Regional Hospital of Koumra, N'Djamena, Chad; Kidza Yvonne Mugerwa, Department of Obstetrics \& Gynaecology, Makerere University, Kampala, Uganda; Anand Tamang, Center for Research on Environment Health and Population Activities, Kathmandu, Nepal; Bukola Fawole, National Institute of Maternal and Child Health, College of Medicine, University of Ibadan, Ibadan, Nigeria; Richard Adanu, School of Public Health, University of Ghana, Accra, Ghana.

Contributors All of the authors participated in the formulation of the study methodology. CRK wrote the first draft of the protocol and the manuscript. ÖT assisted in the editing and writing of the protocol and manuscript. $B G$ and $A M G$ assisted in the editing of the protocol and manuscript. All members of the MCS-A Research Group contributed to the editing of the manuscript.

Funding This study is funded by UNDP/UNFPA/UNICEF/WHO/World Bank Special Programme of Research, Development and Research Training in Human Reproduction (HRP).

Competing interests None declared.

Ethics approval WHO Research Review Panel and Ethics Review Committee.

Provenance and peer review Not commissioned; externally peer reviewed.

Data sharing statement No additional data are available.

Open Access This is an Open Access article distributed in accordance with the Creative Commons Attribution Non Commercial (CC BY-NC 4.0) license, which permits others to distribute, remix, adapt, build upon this work noncommercially, and license their derivative works on different terms, provided the original work is properly cited and the use is non-commercial. See: http:// creativecommons.org/licenses/by-nc/4.0/

\section{REFERENCES}

1. Say L, Chou D, Gemmill A, et al. Global causes of maternal death: a WHO systematic analysis. Lancet Glob Health 2014;2:e323-33.
2. Figa-Talamanca I, Sinnathuray TA, Yusof K, et al. Illegal abortion: an attempt to assess its cost to the health services and its incidence in the community. Int J Health Serv 1986;16:375-89.

3. World Health Organization. Unsafe abortion: global and regional estimates of the incidence of unsafe abortion and associated mortality in 2008. 6th edn. Geneva, Switzerland: World Health Organization, 2011.

4. Singh S, Maddow-Zimet I. Facility-based treatment for medical complications resulting from unsafe pregnancy termination in the developing world, 2012: a review of evidence from 26 countries. BJOG 2016;123:1489-98.

5. Singh S, Prada E, Mirembe F, et al. The incidence of induced abortion in Uganda. Int Fam Plan Perspect 2005;31:183-91.

6. Singh S, Prada E, Kestler E. Induced abortion and unintended pregnancy in Guatemala. Int Fam Plan Perspect 2006;32:136-45.

7. Prada E, Biddlecom A, Singh S. Induced abortion in Colombia: new estimates and change between 1989 and 2008. Int Perspect Sex Reprod Health 2011;37:114-24.

8. Juarez F, Singh S. Incidence of induced abortion by age and state, Mexico, 2009: new estimates using a modified methodology. Int Perspect Sex Reprod Health 2012;38:58-67.

9. Juarez F, Cabigon J, Singh S, et al. The incidence of induced abortion in the Philippines: current level and recent trends. Int Fam Plan Perspect 2005;31:140-9.

10. Fetters $\mathrm{T}$, Vonthanak S, Picardo $\mathrm{C}$, et al. Abortion-related complications in Cambodia. BJOG 2008;115:957-68; discussion 68

11. Gebreselassie H, Fetters T, Singh S, et al. Caring for women with abortion complications in Ethiopia: national estimates and future implications. Int Perspect Sex Reprod Health 2010;36:6-15.

12. Gebreselassie H, Gallo MF, Monyo A, et al. The magnitude of abortion complications in Kenya. BJOG 2005;112:1229-35.

13. Jewkes $\mathrm{R}$, Rees $\mathrm{H}$, Dickson $\mathrm{K}$, et al. The impact of age on the epidemiology of incomplete abortions in South Africa after legislative change. BJOG 2005;112:355-9.

14. Rees $\mathrm{H}$, Katzenellenbogen J, Shabodien R, et al. The epidemiology of incomplete abortion in South Africa. National Incomplete Abortion Reference Group. S Afr Med J 1997;87:432-7.

15. Ziraba AK, Izugbara C, Levandowski BA, et al. Unsafe abortion in Kenya: a cross-sectional study of abortion complication severity and associated factors. BMC Pregnancy Childbirth 2015;15:34.

16. Adler AJ, Filippi V, Thomas SL, et al. Quantifying the global burden of morbidity due to unsafe abortion: magnitude in hospital-based studies and methodological issues. Int J Gynaecol Obstet 2012;118 (Suppl 2):S65-77.

17. Adler AJ, Filippi V, Thomas SL, et al. Incidence of severe acute maternal morbidity associated with abortion: a systematic review. Trop Med Int Health 2012;17:177-90.

18. Prada E, Bankole A, Oladapo OT, et al. Maternal near-miss due to unsafe abortion and associated short-term health and socio-economic consequences in Nigeria. Afr $\mathrm{J}$ Reprod Health 2015;19:52-62.

19. Santana DS, Cecatti JG, Parpinelli MA, et al. Severe maternal morbidity due to abortion prospectively identified in a surveillance network in Brazil. Int J Gynaecol Obstet 2012;119:44-8.

20. Say L, Souza JP, Pattinson RC, et al. Maternal near miss-towards a standard tool for monitoring quality of maternal health care. Best Pract Res Clin Obstet Gynaecol 2009;23:287-96.

21. Souza JP, Gulmezoglu AM, Vogel J, et al. Moving beyond essential interventions for reduction of maternal mortality (the WHO Multicountry Survey on Maternal and Newborn Health): a cross-sectional study. Lancet 2013;381:1747-55.

22. Dragoman M, Sheldon WR, Qureshi Z, et al. Overview of abortion cases with severe maternal outcomes in the WHO Multicountry Survey on Maternal and Newborn Health: a descriptive analysis. BJOG 2014;121(Suppl 1):25-31.

23. Guest P, Ganatra B. The relationship between use of misoprosto and the type and severity of abortion symptoms, 2015. (Personal communication)

24. Naghma-e-Rehan. Cost of the treatment of complications of unsafe abortion in public hospitals. J Pak Med Assoc 2011;61:169-72.

25. Johansson A, Hoa HT, Lap NT, et al. Population policies and reproductive patterns in Vietnam. Lancet 1996;347:1529-32.

26. Banerjee SK, Andersen K. Exploring the pathways of unsafe abortion in Madhya Pradesh, India. Glob Public Health 2012;7:882-96.

27. Rocca $\mathrm{CH}$, Puri M, Dulal B, et al. Unsafe abortion after legalisation in Nepal: a cross-sectional study of women presenting to hospitals. BJOG 2013;120:1075-83.

28. Thonneau P, Matsuda T, Goyaux N, et al. Determinants of maternal deaths in induced abortion complications in Ivory Coast. Contraception 2004;70:319-26. 
29. Adanu RM, Ntumy MN, Tweneboah E. Profile of women with abortion complications in Ghana. Trop Doct 2005;35:139-42.

30. Tuncalp O, Were WM, MacLennan C, et al. Quality of care for pregnant women and newborns - the WHO vision. BJOG 2015;122:1045-9.

31. WHO. Standards for improving quality of maternal and newborn care in health facilities. Geneva, 2016.

32. World Health Organization. Safe abortion: technical and policy guidance for health systems. 2nd edn. Geneva, Switzerland: World Health Organization, 2012.

33. Maternal Health and Safe Motherhood Programme. The prevention and management of unsafe abortion: report of a technical working group, 1993.

34. Ganatra B, Tuncalp O, Johnston HB, et al. From concept to measurement: operationalizing WHO's definition of unsafe abortion. Bull World Health Organ 2014;92:155.

35. Coast E, Murray SF. "These things are dangerous": Understanding induced abortion trajectories in urban Zambia. Soc Sci Med 2016;153:201-9.

36. Rasch V, Sorensen $\mathrm{PH}$, Wang $\mathrm{AR}$, et al. Unsafe abortion in rural Tanzania-the use of traditional medicine from a patient and a provider perspective. BMC Pregnancy Childbirth 2014;14:419.

37. Project Brief: WHO Multi-country Survey on Abortion (WHOMCS-A) 2015. http://who.int/reproductivehealth/projects/13-MCS-ABO.pdf (accessed 22 Aug 2016).

38. Shah A, Faundes A, Machoki M, et al. Methodological considerations in implementing the WHO Global Survey for Monitoring Maternal and Perinatal Health. Bull World Health Organ 2008;86:126-31.

39. Souza JP, Gulmezoglu AM, Carroli G, et al. The world health organization multicountry survey on maternal and newborn health: study protocol. BMC Health Serv Res 2011;11:286.

40. WHO, UNICEF, UNFPA, et al. Trends in maternal mortality: 1990 to 2013. Geneva, Switzerland: WHO, UNICEF, UNFPA, The World Bank and the United Nations Population Division, 2014

41. United Nations Population Division. World Abortion Policies 2013. Secondary World Abortion Policies 2013, 2013. http://www.un.org/ en/development/desa/population/publications/policy/ world-abortion-policies-2013.shtm

42. World Health Organization. Monitoring emergency obstetric care-a handbook. Geneva, Switzerland, 2009.

43. Filippi V. EVA-PMDUP Zambia Near Miss Morbidity. (Unpublished data-Personal communication)

44. Royal College of Obstetrics and Gynaecology. The management of early pregnancy loss. RCOG Green-top Guideline No. 25, 2006.

45. Lessler JT, O'Reilly JM. Mode of interview and reporting of sensitive issues: design and implementation of audio computer-assisted self-interviewing. NIDA Res Monogr 1997;167:366-82.

46. O'Reilly JM, Hubbard ML, Lessler JT, et al. Audio and video computer-assisted self interviewing: preliminary tests of new technologies for data collection. J Off Stat 1994;10:197-214.

47. Metzger DS, Koblin B, Turner C, et al. Randomized controlled trial of audio computer-assisted self-interviewing: utility and acceptability in longitudinal studies. HIVNET Vaccine Preparedness Study Protocol Team. Am J Epidemiol 2000;152:99-106.

48. Mensch BS, Hewett PC, Jones HE, et al. Consistency in women's reports of sensitive behavior in an interview mode experiment, Sao Paulo, Brazil. Int Fam Plan Perspect 2008;34:169-76.

49. Singh S, Remez L, Tartaglione A, eds. Estimating abortion incidence and abortion-related morbidity: a review. New York: Guttmacher Institute, 2010.

50. Singh S, Remez L, Tartaglioin A, eds. Methodologies for estimating abortion incidence and abortion-related morbidity: a review. New York, Paris: Guttmacher Institute and International Union for the Scientific study of Population, 2010.

51. Souza JP, Cecatti JG, Haddad SM, et al. The WHO maternal near-miss approach and the maternal severity index model (MSI): tools for assessing the management of severe maternal morbidity. PLOS ONE 2012;7:e44129.

52. Chou D, Tuncalp O, Firoz T, et al. Constructing maternal morbiditytowards a standard tool to measure and monitor maternal health beyond mortality. BMC Pregnancy Childbirth 2016;16:45.

53. Sarkar S, Seshadri D. Conducting record review studies in clinical practice. J Clin Diagn Res 2014;8:JG01-4. 